\title{
Optimization Model of Network-Level Pavement Maintenance Decision considering User Travel Time and Vehicle Fuel Consumption Costs
}

\author{
Leilei Chen $\mathbb{D}^{1},{ }^{1,2}$ Zepeng Fan $\mathbb{D}^{\mathrm{D}},{ }^{3}$ Pengfei Liu $\mathbb{D}^{\mathrm{D}},{ }^{3}$ and Zhendong Qian ${ }^{1}$ \\ ${ }^{1}$ Key Laboratory of Safety and Risk Management on Transport Infrastructures, Ministry of Transport, \\ Southeast University, Nanjing, China \\ ${ }^{2}$ Intelligent Transportation System Research Center, Southeast University, Nanjing, China \\ ${ }^{3}$ Institute of Highway Engineering, RWTH Aachen University, Aachen, Germany \\ Correspondence should be addressed to Zepeng Fan; fan@isac.rwth-aachen.de
}

Received 10 April 2021; Revised 27 July 2021; Accepted 17 August 2021; Published 27 August 2021

Academic Editor: Adolfo Preciado

Copyright (c) 2021 Leilei Chen et al. This is an open access article distributed under the Creative Commons Attribution License, which permits unrestricted use, distribution, and reproduction in any medium, provided the original work is properly cited.

\begin{abstract}
The maintenance management decisions of network-level asphalt pavements have long been a challenge to highway agencies, and a great amount of factors have been involved. In this study, a network-level optimization method was established by integrating the maintenance benefits into the zero-one programming optimization model. An optimized performance evaluation method of asphalt pavement was proposed which contains 11 different kinds of combinations. The benefit model quantifies the cost savings of user travel time and vehicle fuel consumption to the pavement condition index (PCI) and ride quality index (RQI), respectively. Based on the simplified evaluation method as well as the quantified maintenance benefit model, an optimization model was established by employing the zero-one programming. This optimization model aimed to maximize the improvements/price ratio of pavement maintenance for the whole pavement network. The calculation results present the optimal strategies of maintenance for every road section in the network. The applicability of the newly proposed model was validated by a case study. The methodology developed in this study helps to offer guidelines to highway agencies in managing and making decisions about network-level pavement maintenance.
\end{abstract}

\section{Introduction}

After decades of development, the scale of highway networks in many countries has gradually improved, and they have or are about to face a major shift from the stage of "pavement construction as the main" to "pavement maintenance first." Therefore, pavement maintenance and management issues have attracted more and more attention. In this case, the pavement maintenance management system (PMMS), as a systematic and scientific management and decision support tool, can better optimize and improve the management and decision-making ability of the highway networks. The road in its entire life cycle will get better use performance and higher service levels [1].

The essence of performance evaluation on the pavement service is analysis of the extent to which the pavement performance meets the specified requirements in terms of the investigated pavement condition data. Based on the obtained analysis and evaluation results, the pavement performance can be estimated and the detailed maintenance strategies for the pavement can be planned [2].

The pavement performance evaluation is usually carried out in two aspects, namely, pavement structural and functional evaluations $[3,4]$. The surface distress condition belongs to the structural condition evaluation, which also consists of the structural bearing capacity [5]. The pavement functional evaluation includes driving quality, service level, and safety $[6,7]$. The pavement performance can be evaluated with a single evaluation index or with a comprehensive evaluation index. Specifically, the single evaluation index is mainly for analysis and evaluation of one of the use performances, while multiple single evaluation indexes are 
weighted and integrated as the comprehensive evaluation index for evaluation. In another word, the single evaluation index reflects the condition of a certain performance of the pavement and the comprehensive evaluation index denotes the comprehensive conditions of the pavement service performance [8]. In practice, various parameters are afflicted with large uncertainty. Ignoring the uncertainty may lead to a suboptimal plan adversely affecting the network conditions. Some optimization frameworks for network-level pavement maintenance and rehabilitation planning considering the uncertain nature of pavement deterioration have been developed [9].

The benefit of pavement maintenance is the sum of the direct and the indirect benefits, which arise from the improvement of road conditions by implementing the pavement maintenance on expressways. The benefit calculation of the pavement maintenance projects does not only directly reflect the assessment effectiveness of pavement maintenance but is also an important prerequisite for the proper selection of pavement maintenance methods in the next step. Maintenance managers must allocate their limited budgets among competing alternatives. The absence of simpler decision-making methods exacerbates this issue [10].

Different approaches and perspectives have been developed on the specific indicators and contents of pavement maintenance benefit calculation; for example, the World Bank defined the benefits of road projects into three aspects, namely, benefits arising from the saving in vehicle operating costs, saving in user time costs, and saving in traffic accident reduction. These aspects of benefits are taken account in the specific road project benefit evaluation in many countries, including the United States, United Kingdom, and Japan. Some researchers suggest that not only the short-term, direct, and quantitative economic benefits but also long-term, indirect, and qualitative economic benefits should be considered [11]. Moreover, the economic impact is suggested to be taken into account in the analysis of cost effectiveness [12]. Based on practical experience, the benefits calculation of pavement maintenance projects in China at present mainly considers the benefits of operating costs reduction, the benefits of user travel time saving, the benefits of traffic accidents reduction, and the improvement of the quality of transportation services [13].

Based on the proposed indicators, the problem of finding the best pavement maintenance method can be modeled as a combinatorial optimization problem [14]. Different algorithms have been developed to solve the optimization decision model that can be applied in highway asset management [15-19]. Recently, a binary cuckoo search (BCS) algorithm was implemented to a pavement maintenance management system to solve the optimization problem. The results of a comparison between genetic algorithms (GAs) and the BCS clearly justify the advantages of the search paths underlying the BCS in alleviating premature convergence [20]. Furthermore, several applications using different optimization decision models to improve the overall pavement maintenance solution at the network level can be found. For example, a multiobjective decision-aid tool (MODAT) was developed by testing with data from the
Estradas de Portugal's Pavement Management System. The MODAT uses a multiobjective deterministic section-linked optimization model with different possible goals [21].

However, due to the lack of maintenance experience, the large scale of maintenance requirements, and the shortage of maintenance funds resources, a comprehensive and scientific decision-making method has not yet been established so far. How to establish efficient and scientific pavement maintenance and management methods, improve operational efficiency, meet high-quality pavement maintenance needs, and meet various transportation needs has become important problems encountered in pavement management [22].

This study proposes a network-level pavement maintenance and rehabilitation optimization model considering the costs of user travel time and vehicle fuel consumption. Firstly, the evaluation indicators used in the model were listed. Afterwards, the optimization method of pavement maintenance used in China was briefly introduced. Next, the estimation method of road user costs which was integrated in the optimization method was presented. Based on the theoretical basis, a network-level optimization method considering road user costs was proposed and described in detail. Finally, a case study using the developed optimization method was shown.

\section{Theoretical Basis}

2.1. Evaluation Indicators of Asphalt Pavement. A globally popular pavement performance indicator is the Pavement Condition Index (PCI). It is a numeric index between 0 and 100, which was originally developed by the US Army and later standardized by the ASTM [23]. The PCI reflects the number of the distresses on the pavement and their extent. Therefore, a newly constructed road has a PCI of 100, and as it deteriorates over time and becomes impassable, its PCI approaches 0 . Computing the PCI requires data about several types of distresses and their severity as follows: potholes, fatigue cracking, rutting, block cracking, edge cracking, longitudinal and transverse cracking, patching, shoving, bleeding, polished aggregate, and raveling [24]. The PCI can be determined by the following equation [25]:

$$
\mathrm{PCI}=100-\sum_{i=1}^{n} \sum_{j=1}^{m_{i}} D P_{i j} W_{i j},
$$

where $i$ and $j$ are counters for distress types and severity levels, respectively; $n$ is the total number of observed distress types; $m_{i}$ is the number of severity level for the distress type $i$; $D P_{i j}$ is the deduct value that varies with distress type $i$ and severity $j$; and $W_{i j}$ is an adjustment weight when the pavement with distress type $i$ reaches the severity level $m$.

Pavement roughness is an indispensable criterion in the evaluation of pavement performance, which plays an important role not only in the service life of the pavement but also in the economy of operation and the capacity of vehicles and traffic safety. The Ride Quality Index (RQI) can be used as a standard to evaluate the pavement roughness. The relationship between International Roughness Index (IRI) and 
RQI, according to the Highway Performance Assessment Standards (JTG H20-2007) [25], can be expressed by following equation:

$$
\mathrm{RQI}=\frac{100}{1+a_{0} e^{\left(a_{1} \cdot I R I\right)}},
$$

where $a_{0}$ and $a_{1}$ are the standard parameters with the values of 0.026 and 0.65 , respectively [25].

Skid resistance (or friction) of road surfaces plays a significant role in road safety as the friction between tire, and pavement is a critical contributing factor in reducing potential crashes. This safety performance is also a major criterion for evaluating the service performance of pavements. The structural texture of a pavement greatly affects its skid resistance. The Skid Resistance Index (SRI) of pavement is used to evaluate its skid resistance performance. According to the Highway Performance Assessment Standards (JTG H20-2007) [25], the relationship between the directly measured Sideway Force Coefficient (SFC) and SRI can be described by the following equation:

$$
\mathrm{SRI}=\left(\frac{100-\mathrm{SRI}_{\min }}{1+a_{0} e^{\left(a_{1} \cdot \operatorname{SFC}\right)}}\right)+\mathrm{SRI}_{\min },
$$

where SRI $\min _{\text {in }}$ is the skid resistance limit and $a_{0}$ and $a_{1}$ are the standard parameters with the values of 28.6 and -0.105 , respectively.

Deflection is an effective index to reflect the pavement bearing capacity and structural strength. Measuring deflection has the advantages of being convenient, intuitive, economical, and efficient. According to Highway Performance Assessment Standards (JTG5210-2018) [26], Pavement Structural Strength Index (PSSI) is determined by the allowable pavement deflection, the actually measured pavement deflection, and the model parameters, among which the model parameters are fixed values. When the road structure's own characteristics are determined (such as highway grade and pavement structure type), the pavement's allowable deflection is also uniquely determined [27]. Therefore, the strength of the pavement structure is determined by the measured pavement deflection value as follows:

$$
\text { PSSI }=\frac{100}{1+15.71 e^{-5.19 S S I}},
$$

where SSI is the structure strength index and can be calculated with SSI $=l_{R} / l_{0}$ and $l_{R}$ and $l_{0}$ are the allowed and measured deformations of the asphalt pavement, respectively.

The Pavement Quality Index (PQI) is the overall "score" given to a pavement section with a range from 0 to 100 . The value of 100 represents a road in excellent condition (usually when it is initially constructed). PQI is an output metric that is a function of PCI, RQI, SRI, and PSSI according to [28] as follows:

$$
\mathrm{PQI}=\omega_{\mathrm{PCI}} \mathrm{PCI}+\omega_{\mathrm{RQI}} \mathrm{RQI}+\omega_{\mathrm{SRI}} \mathrm{SRI}+\omega_{\mathrm{PSSI}} \mathrm{PSSI} .
$$

The weight $\omega$ of each index is not fixed but fluctuates in a certain range, which depends on the actual road condition and different maintenance strategies. This can be adjusted appropriately in the recommended range given in the technical specifications, but the values of $0.35,0.35,0.1$, and 0.2 are generally recommended for the four parameters, respectively.

\subsection{Optimization of Pavement Performance Evaluation} Method. Pavement service performance evaluation includes serval indicators such as PCI, RQI, SRI, and PSSI, which is a comprehensive evaluation system. Although PQI can reflect the comprehensive maintenance condition of the pavement, it is necessary to determine the maintenance strategy depending on the specific circumstance, i.e., only using the PQI to develop detailed maintenance strategies is not appropriate. In order to consider the pros and cons of each performance conditions, it requires combining the different pavement performance conditions with different grades for each evaluation indicator, which facilitates the optimization of maintenance decisions.

Current standards in China classify the four indicators (PCI, RQI, SRI, and PSSI) into five levels: excellent, good, fair, poor, and very poor. Therefore, there are in total 625 different combined states, and each state has different maintenance measures, making thousands of possible situations. As a result, the scale of optimization decisionmaking is too complex and is not conducive to solving. It is necessary to analyze and study the combined states of pavement performance to reduce unnecessary combined states and further reduce the scale of optimization decisionmaking. In this study, on basis of analyzing the specific state combinations according to the relevant technical specifications [28], a simplification was adopted by emerging the similar levels to reduce the condition combinations. In particular, the condition levels for the PCI and RQI indexes were adjusted by combining the excellent [90-100] and good [80-90) levels as good [80-100] and also combining fair $[70-80)$ and poor $[60-70)$ as fair $[60-80)$. For the SRI and PSSI indexes, only two condition levels were retained which are sufficient and insufficient. The details for the finalized combinations are shown in Table 1.

It is seen from Table 1 that a total of $3 \times 3 \times 2 \times 2=36$ different condition combinations of asphalt pavement performances after simplification can be summarized. Through this simplification not only the demands of optimization decision-making are fulfilled but also its scale is greatly reduced, which is more beneficial to decisions and solutions. Moreover, it is worth noting that most of these conditions are still of no practical significance. For instance, if the PSSI index is evaluated to be insufficient, structural reinforcement must be conducted regardless of other three indexes. If the SRI index is evaluated to be insufficient but the PSSI index is in the sufficient condition, the anti-skidding layer must be paved to restore the skid resistance regardless of other three indexes. The special requirements on the SRI and PSSI indexes lead to a significant decrease in the effective combinations of pavement condition. In the actual decision-making process, only the combinations with actual significance are necessary for further analysis and the 
TABLE 1: Simplified combinations of the pavement performance condition.

\begin{tabular}{lccc}
\hline & & Verbal rating (numerical rating) & Poor [0-60) \\
PCI & Good [80-100] & Fair [60-80) & Poor [0-60) \\
RQI & Good [80-100] & Fair [60-80) & \\
SRI & Sufficient [62-100] & Insufficient [0-62) & \\
PSSI & Sufficient [80-100] & Insufficient [0-80) & \\
\hline
\end{tabular}

corresponding appropriate maintenance strategies will be selected consequently. Table 2 shows the maintenance strategies for each combination of conditions.

As can be seen in Table 2, only 11 condition combinations are of practical significance for the pavement performance for the maintenance decision optimization problem. Moreover, two combinations (10 and 11) will be eliminated in the later decision optimization due to the demands of the mandatory maintenance strategies. Only the remaining 9 combinations require decision optimizations, and consequently, the scale of decision optimization is reduced.

2.3. Estimation of User Costs. The benefits of expressway pavement maintenance projects can be mainly represented in various related cost savings. The American Association of State Highway and Transportation (AASHO) defines the benefits of pavement maintenance projects as the vehicle travel cost savings, the travel time savings, the traffic accident reduction, and the vehicle exhaust emission reduction. In this study, the user cost savings are defined including the costs of user travel time and the vehicle travel fuel consumption.

2.3.1. Cost Savings in User Travel Time. The user travel time cost is the value that arises due to the existence of an opportunity cost of the time consumed by the vehicle during the trip $[29,30]$. The savings in user travel time cost after implementing pavement maintenance $\Delta T$ can be expressed by the following equation [13]:

$$
\Delta T=\theta \cdot Q \cdot\left(T_{0}-T_{1}\right),
$$

where $\theta$ is the converting coefficient of the travel time value, $Q$ is the traffic volume of the road section, $T_{0}$ is the travel time of a single vehicle before maintenance, and $T_{1}$ is the travel time of a single vehicle after maintenance. The converting coefficient $\theta$ is calculated through $\theta=\mathrm{GDP} /$ (population $\times$ average working time). Calculation of equation (6) requires a functional relationship between the user travel time of the road section and the pavement condition. PCI is then adopted as an indicator for the pavement condition. The functional relationship between $T$ and the PCI can be obtained through tests on a large number of road sections, which is expressed as follows [13]:

$$
T=\alpha \cdot l \cdot(\mathrm{PCI}-\eta)^{\beta}+\frac{l}{v_{0}},
$$

where $l$ is the section distance, $v_{0}$ is the maximal design speed, and $\alpha, \beta$, and $\eta$ are the parameters to be determined.
TABle 2: Condition combinations of the expressway asphalt pavement performance.

\begin{tabular}{lcccc}
\hline Combination & PCI & RQI & SRI & PSSI \\
\hline 1 & Good & Good & Sufficient & Sufficient \\
2 & Fair & Good & Sufficient & Sufficient \\
3 & Poor & Good & Sufficient & Sufficient \\
4 & Good & Fair & Sufficient & Sufficient \\
5 & Fair & Fair & Sufficient & Sufficient \\
6 & Poor & Fair & Sufficient & Sufficient \\
7 & Good & Poor & Sufficient & Sufficient \\
8 & Fair & Poor & Sufficient & Sufficient \\
9 & Poor & Poor & Sufficient & Sufficient \\
10 & & & Insufficient & Sufficient \\
11 & & & & Insufficient \\
\hline
\end{tabular}

Therefore, the savings in user travel time costs after the pavement maintenance can be represented as follows:

$$
\Delta T=\theta \cdot \mathrm{Q} \cdot \alpha \cdot l \cdot\left[\left(\mathrm{PCI}_{0}-\eta\right)^{\beta}-\left(\mathrm{PCI}_{1}-\eta\right)^{\beta}\right]
$$

where $\mathrm{PCI}_{0}$ represents the PCI value of the road before maintenance and $\mathrm{PCI}_{1}$ represents the PCI value of the road after maintenance.

2.3.2. Cost Savings in Vehicle Travel Fuel Consumption. Fuel consumption cost refers to energy expenses consumed by the vehicle in the travel process [31-34]. According to [35], for a minivan, which is selected as the standard vehicle in this study, the relationship among the fuel consumption, the vehicle speed, and the IRI can be expressed by the following equation:

$$
O=0.0040141 \cdot V^{2}-0.56612 \cdot V+25.29872+0.56222 \cdot \text { IRI, }
$$

where $O$ is the fuel consumption $\mathrm{L} / 100 \mathrm{~km}$ and $V$ is the vehicle speed. When assuming that the vehicle is traveling at speed of $80 \mathrm{~km} / \mathrm{h}$, the relationship between the fuel consumption and the IRI can be described by following equation:

$$
O=0.56222 \cdot \operatorname{IRI}+5.69936
$$

Based on equation (10), the cost savings in fuel consumption after pavement maintenance can be expressed as

$$
\Delta O=0.56222 \cdot g \cdot\left(\mathrm{IRI}_{0}-\mathrm{IRI}_{1}\right) \cdot l \cdot \mathrm{Q},
$$

where $g$ is the fuel price, $\mathrm{IRI}_{0}$ represents the IRI value of the road before maintenance, and $\mathrm{IRI}_{1}$ represents the PCI value of the road after maintenance. 


\section{Development of the Optimization Model of Pavement Maintenance at Network Level}

3.1. Problem Statement and Basic Hypotheses. In this study, the selected problem in the pavement maintenance is defined as a highway network consisting of $N_{1}$ roads. According to Section 2.2, there are $N_{2}=11$ combinations of pavement performance conditions. The performance conditions of different sections in the same road may be significantly different, and the proportion of the $i$-th road under the $j$-th combination at a given time is denoted as $d_{i \cdot j}$. A total of $N_{3}$ maintenance strategies are implemented for the highway. To ensure the basic traffic capacity, a minimum value $N_{\mathrm{POI}}$ is generally required for the PQI of the road network after applying the maintenance strategies. Based on this restriction, the maintenance strategies for different roads and performance conditions of road sections are determined to ensure that the performance price ratio of the maintenance is maximized. This means the ratio of road user cost savings to the invested maintenance cost is maximized.

The following hypotheses are proposed in this study for developing the optimization model of pavement maintenance at network level:

(1) In accordance with the requirements of sustainable development, the service level and pavement performance of the road throughout its life cycle do not seriously decline, which means during the planning of the maintenance optimization in the current year, the condition of the service level and pavement performance of the road in the previous year must be considered.

(2) Among the 11 combinations of road performance conditions given in Section 2.2, the 10th and 11th conditions are excluded from the maintenance optimizations due to its regular maintenance strategies. Therefore, in the actual optimization model, $N_{2}$ is reduced from 11 to 9 .

(3) For any road section, only one maintenance strategy is adopted in the current maintenance cycle.

(4) The effects of the increase in traffic volume in the following year on the estimated user costs are neglected.

(5) The implementation of the maintenance does not change the capacity of the road section.

(6) The maintenance measures can reach the required standard values.

3.2. Development of the Optimization Model. According to the maintenance requirements mentioned in earlier section, this study adopts the zero-one integer programming method [36] to construct the maintenance optimization decision model for network-level roads, which are shown in following equations.

The objective function is as follows:

$$
\max \frac{\sum_{i=1}^{N_{1}} \sum_{j=1}^{N_{2}} \sum_{k=1}^{N_{3}}\left(\Delta T_{i \cdot j \cdot k}+\Delta O_{i \cdot j \cdot k}\right)}{\sum_{i=1}^{N_{1}} \sum_{j=1}^{N_{2}} \sum_{k=1}^{N_{3}} C_{i \cdot j \cdot k}} .
$$

The constrains are as follows:

$$
\begin{gathered}
\sum_{k=1}^{N_{3}} x_{i \cdot j \cdot k}=1,\left(i=1,2, \ldots, N_{1} ; j=1,2, \ldots, N_{2} ; k=1,2, \ldots, N_{3}\right), \\
\frac{\sum_{i=1}^{N_{1}} \sum_{j=1}^{N_{2}} \sum_{k=1}^{N_{3}} x_{i \cdot j \cdot k} \cdot l_{i} \cdot d_{i \cdot j} \cdot e_{j \cdot k}}{\sum_{i=1}^{N_{1}} l_{i} \geq N_{P Q I}} \\
x_{i j k}=\{0.1\},
\end{gathered}
$$

where $\Delta T_{i \cdot j \cdot k}$ is the saved user travel time costs after applying the $k$-th maintenance strategy for the $i$-th road section under the $j$-th combination condition, $\Delta O_{i \cdot j \cdot k}$ is the saved fuel consumption from vehicles after applying the $k$-th maintenance strategy for the $i$-th road section under the $j$-th combination condition, $C_{i . j \cdot k}$ is the maintenance cost for applying the $k$-th maintenance strategy for the $i$-th road section under the $j$-th combination condition, $l_{i}$ is the total mileages of the $i$-th road, $d_{i \cdot j}$ is the proportion of the $i$-th road under the $j$-th combination condition, and $e_{j \cdot k}$ is the PQI value after applying the $k$-th maintenance strategy for the road section under the $j$-th combination condition. With equation (13), every road section is ensured a maintenance strategy, and according to the requirements in the actual maintenance of pavements, when a road section is not applied with a special maintenance strategy, the measures for routine road maintenance are still required as necessary. Equation (14) restricts the overall maintenance quality of the road network which guarantees that the average PQI of the road network after maintenance is above 90. Equation (15) defines the 0-1 dummy variable $x_{i \cdot j \cdot k}$ for the zero-one integer programming method used in the current study. The variable $x_{i \cdot j \cdot k}$ has a value of either 0 or 1 . A value of 1 for $x_{i \cdot j \cdot k}$ indicates that the $k$-th maintenance strategy is applied for the $i$-th road section under the $j$-th combination condition or 0 otherwise.

Referring to Section 2.3, $\Delta T_{i \cdot j \cdot k}, \Delta O_{i \cdot j \cdot k}$, and $C_{i \cdot j \cdot k}$ can be determined by following equations:

$$
\begin{aligned}
& \Delta T_{i \cdot j \cdot k}=\theta \cdot Q_{i} \cdot \Delta t_{i \cdot j \cdot k}, \\
& \Delta O_{i \cdot j \cdot k}=Q_{i} \cdot \Delta o_{i \cdot j \cdot k}, \\
& \Delta C_{i \cdot j \cdot k}=x_{i \cdot j \cdot k} \cdot l_{i} \cdot d_{i \cdot j} \cdot p_{i \cdot k},
\end{aligned}
$$

where $Q_{i}$ is the traffic volume of $\mathrm{i}$-th road section, $\Delta t_{i \cdot j \cdot k}$ is the saved user travel time from each vehicle after applying the $k$-th maintenance strategy for the $i$-th road section under the $j$-th combination conditio, $\Delta o_{i \cdot j \cdot k}$ is the saved fuel consumption from each vehicle after applying the $k$-th maintenance strategy for the $i$-th road section under the $j$-th combination condition, and $p_{i \cdot k}$ is the maintenance cost per 
kilometer for the $i$-th road applying the $k$-th maintenance strategy.

$$
\begin{gathered}
\Delta t_{i \cdot j \cdot k} \text { and } \Delta o_{i \cdot j \cdot k} \text { can be obtained through } \\
\Delta t_{i \cdot j \cdot k}=l_{i} \cdot d_{i \cdot j} \cdot t_{j \cdot k}, \\
\Delta o_{i \cdot j \cdot k}=l_{i} \cdot d_{i \cdot j} \cdot g \cdot o_{j \cdot k},
\end{gathered}
$$

where $t_{j \cdot k}$ and $o_{j \cdot k}$ are the saved user travel time and saved fuel consumption from each vehicle after applying the $k$-th maintenance strategy for the $j$-th combination condition in unit kilometer, respectively.

By adopting equations (8) and (11), $t_{j \cdot k}$ and $o_{j \cdot k}$ can be determined with following equations:

$$
\begin{aligned}
t_{j \cdot k} & =\alpha \cdot\left[\left(\mathrm{PCI}_{0}-\eta\right)^{\beta}-\left(\mathrm{PCI}_{1}-\eta\right)^{\beta}\right], \\
o_{j \cdot k} & =0.56222 \cdot\left(\mathrm{IRI}_{0}-\mathrm{IRI}_{1}\right),
\end{aligned}
$$

where $\mathrm{PCI}_{1}$ denotes the pavement condition index of the road under the $j$-th combination condition after applying the $k$-th maintenance strategy, $\mathrm{PCI}_{0}$ represents the pavement condition index of the road under the $j$-th combination condition before applying the $k$-th maintenance strategy, $\mathrm{IRI}_{1}$ expresses the international roughness index of the road under the $j$-th combination condition after applying the $k$-th maintenance strategy, and $\mathrm{IRI}_{0}$ denotes the international roughness index of the road under the $j$-th combination condition before applying the $k$-th maintenance strategy.

\subsection{Case Study}

3.3.1. Background and Input Parameters. Based on operating road sections in Shaanxi, China, the model developed in this study was applied to optimize the maintenance plan decision. Ten expressways were investigated, and all of them are asphalt pavements [37]. The corresponding mileages and traffic volumes are shown in Table 3.

After testing the pavement, the distributions of different combinations of pavement conditions within this network in the current year are presented in Table 4.

By conducting field investigations in different road sections, the costs of five common maintenance strategies for different road sections in this network were determined, as listed in Table 5.

After a comprehensive survey of the current year regarding the gross domestic product (GDP), the population size, and the average working time in Shaanxi Province, the time value conversion coefficient $\theta$ and the current local fuel price $g$ were determined with values of 15.34 and 6.73 Yuan/L, respectively. The value of $N_{\mathrm{PQI}}=90$ is adopted in this case which is suggested by the transport agency.
The determination of the three coefficient matrices $e_{j \cdot k}, t_{j \cdot k}$, and $o_{j \cdot k}$ is required for the application of the model developed in this study. For $e_{j \cdot k}$, PQI can be determined by equation (5), and the averaged values of the pavement performance indicators were adopted in the calculation. In this study, it was assumed that routine maintenance cannot improve the pavement performance evaluation index. The PCI indicator is increased by two levels, and RQI is increased by one level when paving an overlay [38]. For the skid resistance performance index SRI, the optimal level is restored when paving an antiskid layer, conducting the pavement reconstruction or structural reinforcement. For the pavement structural strength index PSSI, the optimal level is restored by conducting a pavement reconstruction or structural reinforcement [38]. For instance, if road section is now in the condition of combination 5, the maintenance strategy of overlay paving is adopted. This means that the PCI and RQI indexes were increased from 70 to 70 to be 95 and 90 , respectively. However, the other two indexes remain unchanged. Then, the PQI of this road after maintenance can be calculated through equation (5) as $\mathrm{PQI}=0.35 \times$ $95+0.35 \times 90+0.1 \times 81+0.2 \times 90=90.85$. Based on these assumptions, the PQI values after applying different maintenance strategies under different combinations are shown in Table 6.

The corresponding coefficient matrices of $t_{j \cdot k}$ and $o_{j \cdot k}$ are shown in Tables 7 and 8, respectively.

\subsubsection{Optimal Maintenance Decision Based on the Calcu-} lation Results. Based on the determined coefficient matrices, the developed model was calculated using lingo software and the optimal maintenance methods were chosen based on the calculation results. The details are shown in Table 9.

It is found from Table 9 that the strategy of structural reinforcement is more preferable than the strategy of pavement rehabilitation for the road sections that are in poor performance. This is expected since the benefits of these two strategies are nearly the same, but the price of rehabilitation is obviously higher than that of structural reinforcement. The method of paving an anti-skid layer is not adopted by the pavements with sufficient skid resistance because this method fails to improve the PCI and RQI indicators. The maximum value of the objective function is 1.73 , indicating that 1.73 Yuan of user cost can be saved per unit of pavement maintenance investment. It is worth noting that the total amount of maintenance cost was not limited in the current model. However, the proposed model can be readily modified to take into account the limit-maintenance-cost cases by introducing an additional constraint on the amount of maintenance cost. 
TABLE 3: Information on mileage and traffic volume of expressway in the road network.

\begin{tabular}{lcccccccccc}
\hline Road number & 1 & 2 & 3 & 4 & 5 & 6 & 7 & 8 & 9 \\
\hline Mileage $(\mathrm{km})$ & 221 & 105 & 141 & 214 & 138 & 87 & 54 & 165 & 69 & 145 \\
Daily traffic volume & 15968 & 25634 & 17964 & 9654 & 14003 & 20658 & 10298 & 18635 & 22384 & 17520 \\
\hline
\end{tabular}

TABLE 4: Distributions of different expressway pavement condition combinations in the road network.

\begin{tabular}{|c|c|c|c|c|c|c|c|c|c|c|}
\hline \multirow{2}{*}{ Combination } & \multicolumn{10}{|c|}{ Road number } \\
\hline & 1 & 2 & 3 & 4 & 5 & 6 & 7 & 8 & 9 & 10 \\
\hline 1 & 0.85 & 0.62 & 1 & 1 & 0.92 & 0.82 & 0.86 & 0.78 & 0.9 & 0.85 \\
\hline 2 & 0.05 & 0.06 & 0 & 0 & 0.08 & 0.11 & 0.06 & 0.09 & 0.1 & 0.1 \\
\hline 3 & 0 & 0 & 0 & 0 & 0 & 0 & 0 & 0 & 0 & 0 \\
\hline 4 & 0.1 & 0.11 & 0 & 0 & 0 & 0.07 & 0.08 & 0.11 & 0 & 0.05 \\
\hline 5 & 0 & 0.06 & 0 & 0 & 0 & 0 & 0 & 0 & 0 & 0 \\
\hline 6 & 0 & 0 & 0 & 0 & 0 & 0 & 0 & 0 & 0 & 0 \\
\hline 7 & 0 & 0.05 & 0 & 0 & 0 & 0 & 0 & 0 & 0 & 0 \\
\hline 8 & 0 & 0.06 & 0 & 0 & 0 & 0 & 0 & 0 & 0 & 0 \\
\hline 9 & 0 & 0 & 0 & 0 & 0 & 0 & 0 & 0 & 0 & 0 \\
\hline 10 & 0 & 0.04 & 0 & 0 & 0 & 0 & 0 & 0.02 & 0 & 0 \\
\hline 11 & 0 & 0 & 0 & 0 & 0 & 0 & 0 & 0 & 0 & 0 \\
\hline
\end{tabular}

TABLE 5: Cost of different road sections with different maintenance strategies $\left(10000 \mathrm{Yuan} / \mathrm{m}^{2}\right)$.

\begin{tabular}{|c|c|c|c|c|c|c|c|c|c|c|}
\hline & \multicolumn{10}{|c|}{ Road number } \\
\hline & 1 & 2 & 3 & 4 & 5 & 6 & 7 & 8 & 9 & 10 \\
\hline Routine maintenance & 5 & 5 & 4.5 & 4.5 & 5 & 4 & 4.5 & 4 & 5 & 4 \\
\hline Paving of overlay & 20 & 20 & 18 & 18 & 20 & 15 & 18 & 15 & 20 & 15 \\
\hline Paving of anti-skid layer & 15 & 15 & 13 & 13 & 15 & 12 & 13 & 12 & 15 & 12 \\
\hline Pavement reconstruction & 50 & 50 & 47 & 47 & 50 & 42 & 47 & 42 & 50 & 42 \\
\hline Structural reinforcement & 40 & 40 & 36 & 36 & 40 & 32 & 36 & 32 & 40 & 32 \\
\hline
\end{tabular}

TABLE 6: PQI values after applying different maintenance strategies under different combinations.

\begin{tabular}{lccccc}
\hline Combination & Routine maintenance & Paving of overlay & Paving of anti-skid layer & Pavement reconstruction & Structural reinforcement \\
\hline 1 & 89.1 & 92.6 & 90.5 & 95 & 95 \\
2 & 82.1 & 92.6 & 83.5 & 95 & 95 \\
3 & 68.1 & 90.85 & 69.5 & 95 & 95 \\
4 & 82.1 & 90.85 & 83.5 & 95 & 95 \\
5 & 75.1 & 90.85 & 66.5 & 95 & 95 \\
6 & 61.1 & 89.1 & 62.5 & 95 & 95 \\
7 & 68.1 & 83.85 & 62.5 & 95 & 95 \\
8 & 61.1 & 83.85 & 48.5 & 95 & 95 \\
9 & 47.1 & 82.1 & & & 95 \\
\hline
\end{tabular}

TABLe 7: The $t_{j \cdot k}$ matrix used in the developed model.

\begin{tabular}{lccccc}
\hline Combination & Routine maintenance & Paving of overlay & Paving of anti-skid layer & Pavement reconstruction & Structural reinforcement \\
\hline 1 & 0 & 0.0000015 & 0 & 0.0000014530 & 0.0000014530 \\
2 & 0 & 0.0000088 & 0 & 0.0000088458 & 0.0000088458 \\
3 & 0 & 0.0001232 & 0 & 0.0001246440 & 0.0001246440 \\
4 & 0 & 0.0000015 & 0 & 0.0000014530 & 0.0000014530 \\
5 & 0 & 0.0000088 & 0 & 0.0000088458 & 0.0000088458 \\
6 & 0 & 0.0001232 & 0 & 0.0001246440 & 0.0001246440 \\
7 & 0 & 0.0000015 & 0 & 0.0000014530 & 0.0000014530 \\
8 & 0 & 0.0000088 & 0 & 0.0000088458 & 0.0000088458 \\
9 & 0 & 0.0001232 & 0 & & 0.0001246440 \\
\hline
\end{tabular}


TABle 8: The $o_{j . k}$ matrix used in the developed model.

\begin{tabular}{lccccc}
\hline Combination & Routine maintenance & Paving of overlay & Paving of anti-skid layer & Pavement reconstruction & Structural reinforcement \\
\hline 1 & 0 & 0.6463060 & 0 & 0.6463059708 & 0.6463059708 \\
2 & 0 & 0.6463060 & 0 & 0.6463059708 & 0.6463059708 \\
3 & 0 & 0.6463060 & 0 & 0.6463059708 & 0.6463059708 \\
4 & 0 & 1.1676243 & 0 & 1.8139302766 & 1.8139302766 \\
5 & 0 & 1.1676243 & 0 & 1.8139302766 & 1.8139302766 \\
6 & 0 & 1.1676243 & 0 & 3.2939468546 & 3.8139302766 \\
7 & 0 & 1.4657549 & 0 & 3.2939468546 & 3.293946854646 \\
8 & 0 & 1.4657549 & 0 & 3.2939468546 & 3.2939468546 \\
9 & 0 & 1.4657549 & 0 & & \\
\hline
\end{tabular}

TABle 9: Optimal maintenance methods on the different road sections under different combinations.

\begin{tabular}{lcccccccccc}
\hline \multirow{2}{*}{ Combination } & \multicolumn{10}{c}{ Road number } \\
& 1 & 2 & 3 & 4 & 5 & 6 & 7 & 8 & 9 & 10 \\
\hline 1 & I & II & II & I & I & II & I & II & II & II \\
2 & I & II & & & I & II & I & II & II & II \\
3 & & & & & & & & & & \\
4 & II & V & & & & V & II & V & & II \\
5 & & V & & & & & & & & \\
6 & & & & & & & & & & \\
7 & & V & & & & & & & & \\
8 & & V & & & & & & & & \\
9 & & & & & & & & & & \\
10 & & III & & & & & III & & \\
11 & & & & & & &
\end{tabular}

I denotes the routine maintain, II represents paving of overlay, III indicates the paving of anti-skid layer, IV refers to the pavement rehabilitation, and $\mathrm{V}$ denotes the structural reinforcement.

\section{Conclusions and Outlook}

This study presents the development and implementation of a network-level optimization method within a pavement management information system. The network-level optimization method integrates the estimation method of road user costs including the user travel time and vehicle fuel consumption, and it provides the highway agencies the ability to making better and more reasonable managing and making decisions about network-level pavement maintenance. The major conclusions of this study are as follows.

The performance evaluation method of asphalt pavement was optimized to include 11 different combinations. The benefit model of asphalt pavement maintenance was established by taking the user cost into account, which includes cost savings in both user travel time and vehicle fuel consumption. Based on the simplified evaluation method of pavement performance as well as the quantified estimation method of user cost, an optimization model was established. The new model is capable of (1) maximizing the improvements/price ratio of pavement maintenance for the given road network and (2) determining the corresponding strategy of maintenance for any road section in the road network. The optimization model developed in the current study paves the path for the integrating of user travel time and vehicle fuel consumption costs into the application network-level road maintenance decision. It is worth noting that the model established here can be readily extended for the investigation of more complicated road networks or consideration of other impact factors.

For future research, more components of user cost (e.g., vehicle tire wearing cost and vehicle safety cost) as well as the environment costs including exhaust emission pollution cost and traffic noise pollution cost should be incorporated into the optimization model. The effects of the increase in traffic volume in the following year on the estimation of user costs should be considered.

\section{Data Availability}

The data used to support the findings of this study are included within the article.

\section{Conflicts of Interest}

The authors declare that they have no conflicts of interest.

\section{Acknowledgments}

The financial support from the Fundamental Research Funds for the Central Universities (Grant Project No. $2242021 \mathrm{k} 30034)$ is gratefully acknowledged.

\section{References}

[1] G. Loprencipe, A. Pantuso, and P. Di Mascio, "Sustainable pavement management system in urban areas considering the vehicle operating costs," Sustainability, vol. 9, no. 3, p. 453, 2017.

[2] P. Liu, F. Otto, D. Wang, M. Oeser, and H. Balck, "Measurement and evaluation on deterioration of asphalt pavements by geophones," Measurement, vol. 109, pp. 223-232, 2017.

[3] O. Elbagalati, M. Elseifi, K. Gaspard, and Z. Zhang, "Development of the pavement structural health index based on falling weight deflectometer testing," International Journal of Pavement Engineering, vol. 19, no. 1, pp. 1-8, 2018.

[4] X. Cai, D. Wang, and J. Yu, "Evaluation of the functional performance of paving materials based on the driving wheel pavement analyzer," Applied Sciences, vol. 10, no. 7, p. 2410, 2020.

[5] P. Liu, D. Wang, F. Otto, J. Hu, and M. Oeser, "Application of semi-analytical finite element method to evaluate asphalt pavement bearing capacity," International Journal of Pavement Engineering, vol. 19, no. 6, pp. 479-488, 2018. 
[6] J. Zhang, M. Wang, D. Wang, X. Li, B. Song, and P. Liu, "Feasibility study on measurement of a physiological index value with an electrocardiogram tester to evaluate the pavement evenness and driving comfort," Measurement, vol. 117, pp. 1-7, 2018.

[7] P. Liu, V. Ravee, D. Wang, and M. Oeser, "Study of the influence of pavement unevenness on the mechanical response of asphalt pavement by means of the finite element method," Journal of Traffic and Transportation Engineering, vol. 5, no. 3, pp. 169-180, 2018.

[8] K. Kalita and P. Rajbongshi, "Variability characterisation of input parameters in pavement performance evaluation," Road Materials and Pavement Design, vol. 16, no. 1, pp. 172-185, 2015.

[9] A. Fani, A. Golroo, S. Ali Mirhassani, and A. H. Gandomi, "Pavement maintenance and rehabilitation planning optimisation under budget and pavement deterioration uncertainty," International Journal of Pavement Engineering, pp. 1-11, 2020.

[10] M. Jesus, S. Akyildiz, D. R. Bish, and D. A. Krueger, "Networklevel optimization of pavement maintenance renewal strategies," Advanced Engineering Informatics, vol. 25, no. 4, pp. 699-712, 2011.

[11] Z. Li, Multicriteria Highway Programming Incorporating Risk and Uncertainty: A Methodology for Highway Asset Management System, Purdue University, West Lafayette, IN, USA, 2004.

[12] Y. Pan, Mechanism of Pavement Management System, China Communications Press, Beijing, China, 1998.

[13] X. Mao, Research on improvement of expressway pavement maintenance decision, ph.D. Thesis, Chang'an Universtiy, Xi'an, China, 2015.

[14] J. Santos, A. Ferreira, and G. Flintsch, "An adaptive hybrid genetic algorithm for pavement management," International Journal of Pavement Engineering, vol. 20, no. 3, pp. 266-286, 2019.

[15] C. Blum and A. Roli, "Metaheuristics in combinatorial optimization," ACM Computing Surveys, vol. 35, no. 3, pp. 268-308, 2003

[16] N. R. Tayebi, F. M. Nejad, and M. Mola, "Comparison between GA and PSO in analyzing pavement management activities," Journal of Transportation Engineering, vol. 140, no. 1, pp. 99-104, 2014.

[17] V. Yepes, C. Torres-Machi, A. Chamorro, and E. Pellicer, "Optimal pavement maintenance programs based on a hybrid greedy randomized adaptive search procedure algorithm," Journal of Civil Engineering and Management, vol. 22, no. 4, pp. 540-550, 2016.

[18] Z. Li and K. C. Sinha, "Methodology for multicriteria decision making in highway asset management," Transportation Research Record: Journal of the Transportation Research Board, vol. 1885, no. 1, pp. 79-87, 2004.

[19] J. C. Chu and K.-H. Huang, "Mathematical programming framework for modeling and comparing network-level pavement maintenance strategies," Transportation Research Part B: Methodological, vol. 109, pp. 1-25, 2018.

[20] F. Xiao, S. Yang, J. Cheng, M. Hou, and C. Wang, "A binary cuckoo search for combinatorial optimization in multiyear pavement maintenance programs," Advances in Civil Engineering, vol. 2020, Article ID 8851325, 12 pages, 2020.

[21] S. Meneses and A. Ferreira, "Pavement maintenance programming considering two objectives: maintenance costs and user costs," International Journal of Pavement Engineering, vol. 14, no. 2, pp. 206-221, 2013.
[22] S. A. Hosseini and O. Smadi, "How prediction accuracy can affect the decision-making process in pavement management system," Infrastructure, vol. 6, no. 2, p. 28, 2021.

[23] D. Astm, Standard Practice for Roads and Parking Lots Pavement Condition index Surveys, Standard Publucation, London, UK, 2009.

[24] S. M. Piryonesi and T. E. El-Diraby, "Examining the relationship between two road performance indicators: pavement condition index and international roughness index," Transportation Geotechnics, vol. 26, Article ID 100441, 2021.

[25] M. China, Highway Performance Assessment Standards: JTG H20-2007, Standard Prees of China, Beijing, China, 2007.

[26] M. China, Highway Performance Assessment Standards: JTG 5210-2018, Standard Prees of China, Beijing, China, 20018.

[27] Q. Yang and S. Zhou, "Theoretical analysis of pavement deflection response sensitivity under steady-state excitation," International Journal of Transportation Science and Technology, 2020.

[28] M. China, Technical Specifications for Maintenance of Highway Asphalt Pavement: JTJ 073, Standard Prees of China, Beijing, China, 2001.

[29] G. S. Becker, "A theory of the allocation of time," Economic Journal, vol. 75, pp. 93-517, 1965.

[30] W. Mark, "A review of British evidence on time and service quality valuations," Transportation Research Part E, vol. 37, pp. 107-128, 2001.

[31] G. Scora and M. Barth, Comprehensive Modal Emissions Model (CMEM), Version 3.01. User Guide, Centre for environmental research and technology. University of California, Riverside, CA, USA, 2006.

[32] K. T. Chau, Y. S. Wong, and C. C. Chan, "Evsim - a PC-based simulation tool for an electric vehicle technology course," International Journal of Electrical Engineering Education, vol. 37, no. 2, pp. 167-179, 2000.

[33] H. C. Frey, N. M. Rouphail, H. Zhai, T. L. Farias, and G. A. Gonçalves, "Comparing real-world fuel consumption for diesel- and hydrogen-fueled transit buses and implication for emissions," Transportation Research Part D: Transport and Environment, vol. 12, no. 4, pp. 281-291, 2007.

[34] C. M. Silva, T. L. Farias, H. C. Frey, and N. M. Rouphail, "Evaluation of numerical models for simulation of real-world hot-stabilized fuel consumption and emissions of gasoline light-duty vehicles," Transportation Research Part D: Transport and Environment, vol. 11, no. 5, pp. 377-385, 2006.

[35] Y. Zhou and H. R. Zhang, "Research on relation between of surface characteristics and fuel consumption," Highways, vol. 1, no. 1, pp. 30-36, 2005.

[36] M. Wang, Y. Wang, X. Gao, and C. Sweetapple, "Combination and placement of sustainable drainage system devices based on zero-one integer programming and schemes sampling," Journal of Environmental Management, vol. 238, pp. 59-63, 2019.

[37] S. Cao, Research on Network Maintenance Decision of Expressway Asphalt Pavement Based on Matter Element Analysis Master Thesis, Chang'an University, Xi'an, China, 2018.

[38] Y. Pan, P. Zheng, A. Zheng, S. Bao, and Z. Wu, "Analysis model of investment for major and intermediate maintenance of asphalt pavement," China Journal of Highway and Transport, vol. 5, no. 3, pp. 14-21, 1992. 